\title{
ON EXISTENCE OF OSCILLATORY SOLUTIONS OF A SECOND-ORDER NONLINEAR DIFFERENTIAL EQUATION
}

\author{
James S. W. Wong \\ Dedicated to F. V. Atkinson
}

\begin{abstract}
This paper studies the existence of oscillatory solutions of the secondorder nonlinear differential equation $y^{\prime \prime}+y \exp \left(|y|^{m}-x\right)=0$ on $[0, \infty)$ for $m>0$. Our result proves a conjecture by F.V. Atkinson that this equation has oscillatory solutions for $m \geq 2$. This, together with the original results of Atkinson and Chen proving nonoscillation of this equation for $0<m<2$, completely settles the question of existence of oscillatory solutions.
\end{abstract}

\section{Introduction}

Consider the second-order nonlinear differential equation on the semi-infinite axis $[0, \infty)$

$$
y^{\prime \prime}+y \exp \left(|y|^{m}-x\right)=0, \quad m>0 .
$$

Equation (1) arises in connection with radial solutions of the partial differential equations $[2,3]$

$$
\Delta u+f(u)=0, \quad u \in C^{2}\left(\mathbf{R}^{2}\right),
$$

where $\Delta$ denotes the Laplacian, and $f(u)$ behaves like $\exp \left(|u|^{m}\right)$. It was pointed out in [3] that $f(u)=\exp \left(u^{2}\right)$ was well known to be a borderline case.

It is known from that literature, see $[6,8]$, that solutions to (1) with arbitrary initial data can be continued throughout the entire half real axis. A solution $y(x)$ is said to be oscillatory if it has arbitrarily large zeros, i.e., for each $x_{0}>0$, there exists an $x_{1} \geq x_{0}$ such that $y\left(x_{1}\right)=0$. Equation (1) is called oscillatory if all solutions are oscillatory and is called nonoscillatory otherwise. In general, (1) may possess oscillatory and nonoscillatory solutions simultaneously.

Equation (1) is a special case of the more general second-order nonlinear differential equation

$$
y^{\prime \prime}+y F\left(y^{2}, x\right)=0, \quad 0<x<\infty,
$$

where $F(r, x)$ is continuous in $r$ on $[0, \infty)$ for every $0<x<\infty$ and piecewise continuous in $x$ on $[0, \infty)$ for every $r \geq 0$. Equation (3) first was introduced by Nehari [13]

Received February 26, 1997, revised June 25, 1997.

1991 Mathematics Subject Classification: 34C10, 34C15.

Key words and phrases: ordinary differential equations, second order, nonlinear, oscillatory solutions. 
with a view to generalize oscillation results of Atkinson [1] for the Emden-Fowler equation, see [15],

$$
y^{\prime \prime}+q(x) y|y|^{\gamma-1}=0, \quad \gamma>1,
$$

where $q(x)$ is continuous in $x$ on $[0, \infty)$. Assume that $F(r, x)$ is sufficiently smooth in both $r$ and $x$, e.g., differentiable with respect to $r$ and $x$, and that $F(r, x)$ is nonnegative. Nehari [13] then proved that (3) has a nonoscillatory solution if and only if

$$
\int^{\infty} x F(c, x) d x<\infty
$$

for some constant $c>0$. Since $x e^{-x} \exp \left(c^{m}\right) \in L^{1}[0, \infty)$ for $m>0$, it follows that (1) always has nonoscillatory solutions. Nehari's proof is in fact an adaptation of an original proof given by Atkinson [1] for the more special equation (4). Thus, equation (1) cannot have all of its solutions oscillatory for any $m>0$. The question remains for what values of $m$ does equation (1) have oscillatory solutions.

Atkinson and Chen [4] proved that (1) is nonoscillatory for $0<m<2$, i.e., it cannot have any oscillatory solution. Recently, Hastings and McLeod [9] showed that (1) with $m=2$ has oscillatory solutions. The purpose of this paper firstly is to prove that for $m>2,(1)$ also has oscillatory solutions. Secondly, we present an alternative and simpler proof of the result of Hastings and McLeod. The proof given in [9] is based upon phase plane analysis of a perturbed 2-dimensional autonomous system which is shown to be equivalent to (1). By identifying limit cycles of the unperturbed autonomous system, the authors succeeded in showing the existence of solutions of (1) which have these limit cycles as their limiting sets. Hence, these solutions must be oscillatory. As phase-plane analysis for autonomous systems can be cumbersome at times, our approach is more direct, and the resulting proof is considerably shorter.

Finally, we return to the proof by Atkinson and Chen [4] for the case when $0<$ $m<2$. To prove nonoscillation, their proof is based upon an asymptotic estimate of the growth of zeros of any oscillatory solutions when they exist. In fact, let $\left\{s_{n}\right\}$ be the sequence of consecutive zeros of $y^{\prime}(x)$. It was shown that $s_{n} \sim K n^{\mu}$ where $\mu=m /(m-1)$ and $K$ is some suitable constant. Their proof used rather clever inequalities and finally appealed to a nonoscillation result of Coffman and Wong [6] which also involves complicated identities. Our proof for nonoscillation as given in this paper is more direct and appreciably simpler.

\section{Existence of oscillatory solutions, $m>2$}

We consider a more general equation than (1) in the form

$$
y^{\prime \prime}+a(x) f(y)=0, \quad 0<x<\infty .
$$

Here $a(x)$ is assumed to be piecewise continuous in $x$, and $f(y)$ is odd, i.e., $f(-y)=$ $-f(y)$, and superlinear, i.e., $u^{-1} f(u)$ is non-decreasing for $u>0$. Equation (6) covers the special equation (1) and is a special case of the more general equation (3).

Theorems on the existence of oscillatory solutions which do not preclude existence of nonoscillatory solutions were first given by Jasny [11] and Kurzweil [12] for the Emden-Fowler equation (4). Their result later was extended to the more general equation (3) in an earlier paper by Coffman and the author [5]. Unfortunately, the result as stated in [5] (and its further generalization as given in [6]) is not directly 
applicable to the study of (1). In this section, we shall borrow the techniques in our earlier work $[5,6]$ and prove a result which would establish the existence of oscillatory solutions of (1) when $m>2$. Our first result is

Theorem 1. Suppose that (a) there exist constants $x_{0}, M, c>0$ such that

$$
x^{2} f(u) u^{-1} a(x) \geq \frac{1}{4}+c
$$

where $u^{2} \geq M^{2} x$ and $x \geq x_{0}$, and (b) for all $\alpha>0, x a(x) F\left(\alpha x^{1 / 2}\right)$ is nondecreasing for $x \geq x_{0}$ where $F(u)=\int_{0}^{u} f(v) d v$. Then every solution of (6) with a zero is oscillatory.

We prove the theorem with the aid of the following lemmas.

Lemma 1. Let $y(x)$ be a nonoscillatory solution of (6), then

$$
\liminf _{x \rightarrow \infty} \frac{x^{2} a(x) f(y(x))}{y(x)} \leq \frac{1}{4} .
$$

Proof. Assume that (8) is false, it follows that $y(x)$ satisfies the linear equation $z^{\prime \prime}+$ $q(x) z=0$ where

$$
q(x)=\frac{a(x) f(y(x))}{y(x)} \geq \frac{1+\varepsilon}{4 x^{2}}
$$

for some $\varepsilon>0$. By Sturm's Comparison Theorem, $z^{\prime \prime}+q(x) z=0$ is oscillatory, which contradicts the assumption that $y(x)$ is nonoscillatory.

Lemma 2. Let $y(x)$ be a solution of (6). Define

$$
H(y(x))=x y^{\prime 2}+2 x a(x) F(y)-y y^{\prime} .
$$

If $x a(x) F\left(\alpha x^{1 / 2}\right)$ is non-decreasing for $x \geq x_{0}$, then $\frac{d}{d x} H(y(x)) \geq 0$ for $x \geq x_{0}$.

Proof. Write $H(x)=H(y(x))$ for short. Use (6) to compute

$$
\begin{aligned}
H^{\prime}(x) & =2(x a(x))^{\prime} F(y)+a(x) y f(y) \\
& =\left.2 \frac{\partial}{\partial \sigma}\left(\sigma a(\sigma) F\left(\frac{y(t) \sqrt{\sigma}}{\sqrt{t}}\right)\right)\right|_{\sigma=t},
\end{aligned}
$$

which is non-negative since $x a(x) F(\alpha \sqrt{x})$ is non-decreasing in $x$ for every $\alpha>0$ and $F(u)$ is an even function of $u$.

Lemma 3. Let $y(x)$ be a nonoscillatory solution of (6). Denote $\varphi(x)=\sqrt{x} y^{\prime}-$ $\frac{1}{2} y / \sqrt{x}$. If $\varphi(x)$ is eventually of one sign, then

$$
\lim _{x \rightarrow \infty} \varphi(x)=0 \text {. }
$$

Proof. Note that $(y / \sqrt{x})^{\prime}=\varphi(x) / x$, so if $\varphi(x)$ is eventually of one sign, then we have

$$
J=\int^{\infty}\left|\left(\frac{y}{\sqrt{x}}\right)^{\prime}\right| d x=\left|\int^{\infty}\left(\frac{y}{\sqrt{x}}\right)^{\prime} d x\right| .
$$

If $\varphi(x) \leq 0$, then the definite integral $J$ defined above is finite. Otherwise, when $\varphi(x) \geq 0$, we note that

$$
\int_{x_{1}}^{x}\left(\frac{y}{\sqrt{x}}\right)^{\prime} d s=\frac{y(x)}{\sqrt{x}}-\frac{y\left(x_{1}\right)}{\sqrt{x_{1}}}
$$


Assume that there exists an $x_{1} \geq x_{0}$ so that $y\left(x_{1}\right)>M \sqrt{x_{1}}$, and since $\varphi(x) \geq 0$, then $y(x)>M \sqrt{x}$ for all $x \geq x_{1}$. This implies by (7) that

$$
\frac{x^{2} a(x) f(y(x))}{y(x)} \geq \frac{1}{4}+c
$$

which contradicts (8) because $y(x)$ is nonoscillatory. It then follows that $0<y(x) \leq$ $M \sqrt{x}$ for $x \geq x_{0}$. To prove (11), let $\lim \sup _{t \rightarrow \infty} \varphi(x)=Q>0$. Choose $A$ such that

$$
\int_{A}^{\infty}\left|\left(\frac{y}{\sqrt{x}}\right)^{\prime}\right| d x<\frac{Q^{2}}{8 M} .
$$

Also choose $x_{0}, x_{1}$ such that $x_{1}>x_{0} \geq A, \varphi\left(x_{1}\right) \geq Q / 2$ and

$$
\int_{x_{0}}^{x_{1}} \frac{d x}{x}=\frac{Q}{M}
$$

Observe that

$$
\varphi^{\prime}(x)=\sqrt{x} y^{\prime \prime}+\frac{y}{4 x^{2 / 3}} \leq \frac{M}{4 x},
$$

from which we can estimate that for $x_{0} \leq x \leq x_{1}$,

$$
\varphi(x)-\varphi\left(x_{1}\right)=-\int_{x}^{x_{1}} \varphi^{\prime}(x) d s \geq-\frac{M}{4} \int_{x}^{x_{1}} \frac{d s}{s} \geq-\frac{Q}{4} .
$$

Using (12), we find

$$
\begin{aligned}
\int_{x_{0}}^{x_{1}}\left(\frac{y}{\sqrt{x}}\right)^{\prime} d x & =\int_{x_{0}}^{x_{1}} \frac{\varphi(x)}{x} d x \\
& \geq\left(\varphi\left(x_{1}\right)-\frac{Q}{4}\right) \int_{x_{0}}^{x_{1}} \frac{d x}{x} \\
& \geq\left(\frac{Q}{2}-\frac{Q}{4}\right) \frac{Q}{M} \\
& =\frac{Q^{2}}{4 M},
\end{aligned}
$$

which contradicts the fact that $\int_{A}^{\infty}(y / \sqrt{x})^{\prime}<Q^{2} / 8 M$. Thus

$$
\lim _{x \rightarrow \infty} \varphi(x)=0 \text {. }
$$

We now are ready to complete the proof of Theorem 1. Suppose that $y(x)$ is a nonoscillatory solution. Since $-y(x)$ is also a solution of $(6)$ whenever $y(x)$ is, we may assume that $y(x)$ is positive, say, for $x \geq x_{0}$. Consider $\varphi(x)$ in two mutually exclusive cases: one when $\varphi(x)$ is oscillatory, i.e., there exists a sequence $\left\{\xi_{n}: \xi_{n} \geq x_{0}, \varphi\left(\xi_{n}\right)=\right.$ $0\}$ and $\xi_{n} \rightarrow \infty$ as $n \rightarrow \infty$, and its alternative when $\varphi(x)$ is eventually of one sign. In the first case, we further may extract a subsequence from $\left\{\xi_{n}\right\}$ which we denote again by $\left\{\xi_{n}\right\}$ so that $\left\{\xi_{n}: \xi_{n} \geq x_{0}, \varphi\left(\xi_{n}\right)=0, \varphi^{\prime}\left(\xi_{n}\right) \geq 0\right\}$ and observe that

$$
0 \leq \varphi^{\prime}\left(\xi_{n}\right) \sqrt{\xi_{n}}=-\xi_{n} a\left(\xi_{n}\right) f\left(y\left(\xi_{n}\right)\right)+\frac{y\left(\xi_{n}\right)}{4 \xi_{n}} .
$$


On the other hand, if $\varphi(x)$ is not oscillatory, hence eventually of one sign, we have (11). If $y(x)$ is non-oscillatory, then by (8) there also exists a sequence $\left\{\xi_{n}\right\}$ so that (13) holds.

We now rewrite $H(x)$ as

$$
\begin{aligned}
H(x) & =x\left(y^{\prime}-\frac{y}{2 x}\right)^{2}+2 x a(x) F(y)-\frac{y^{2}}{4 x} \\
& =\varphi(x)^{2}+2 x a(x) F(y)-\frac{y^{2}}{4 x} .
\end{aligned}
$$

Since $f(y)$ is assumed to be superlinear, we note for $y>0$ that

$$
F(y)=\int_{0}^{y} f(u) d u \leq \frac{f(y)}{y} \int_{0}^{y} u d u
$$

which implies

$$
2 x a(x) F(y) \leq x a(x) y f(y) .
$$

Using (15) in (14) and evaluating $H(x)$ at sequence $\left\{\xi_{n}\right\}$ where (13) holds, we find

$$
\lim _{n \rightarrow \infty} H\left(y\left(\xi_{n}\right)\right) \leq 0
$$

By Lemma 2, we conclude that

$$
\lim _{x \rightarrow \infty} H(y(x))=0
$$

for every nonoscillatory solution $y(x)$. Let $y(x)$ be a solution with a zero at $x_{0}$ and $y^{\prime}\left(x_{0}\right) \neq 0$, so that $H\left(y\left(x_{0}\right)\right)=x_{0} y^{\prime 2}\left(x_{0}\right)>0$. Since $H(y(x))$ is non-decreasing in $x$, (16) yields the desired contradiction. Hence, $y(x)$ must be oscillatory.

To apply Theorem 1 to equation (1), we have $a(x)=e^{-x}$ and $f(y)=y \exp \left(|y|^{m}\right)$, $m>2$. Clearly, $f(y)$ is odd and superlinear in $u$. Choosing $M=1, c>0$ in (7), we find

$$
\begin{aligned}
x^{2} f(u) u^{-1} a(x) & =x^{2} \exp \left(|u|^{m}-x\right) \\
& \geq x^{2} \exp \left(x^{m / 2}-x\right) \geq \frac{1}{4}+c,
\end{aligned}
$$

which clearly is satisfied if $x$ is sufficiently large and $m>2$. To show that $x a(x) F(\alpha \sqrt{x})$ is non-decreasing, we note that for large values of $|y|, F(y)$ behaves as

$$
\begin{aligned}
F(|y|) & =\int_{0}^{|y|} s \exp s^{m} d s \\
& =\frac{1}{m}|y|^{2-m} \exp |y|^{m}\left\{1+O\left(|y|^{-m}\right)\right\} .
\end{aligned}
$$

We now differentiate $x a(x) F(\alpha \sqrt{x})$ and obtain by (17)

$$
\begin{aligned}
\frac{d}{d x} x e^{-x} F(\alpha \sqrt{x}) & =\frac{x e^{-x} f(\alpha \sqrt{x}) \alpha}{2 \sqrt{x}}+\left(e^{-x}-x e^{-x}\right) F(\alpha \sqrt{x}) \\
& =e^{-x} \exp |\alpha \sqrt{x}|^{m}\left\{\frac{\alpha^{2}}{2} x+(1-x) \frac{\alpha^{2-m}}{m} x^{1-\frac{m}{2}}+O\left(|x|^{-m / 2}\right)\right\} .
\end{aligned}
$$

Note that the leading negative term $1 / m\left(\alpha^{2-m} x^{2-m / 2}\right)$ in (18) is dominated by the leading positive term $\left(\alpha^{2} / 2\right) x$ when $m>2$. Thus $x e^{-x} F(\alpha \sqrt{x})$ is non-decreasing for 
sufficiently large $x$. We now conclude from Theorem 1 that equation (1) has oscillatory solutions for $m>2$. In fact, Theorem 1 also proves that every solution of (1) with a zero is oscillatory when $m>2$. This leads naturally to the borderline case when $m=2$, and the question whether (1) also has oscillatory solutions in this case. In the next section, we shall answer the question in the affirmative.

\section{The borderline case when $m=2$}

In this section, we consider the borderline case when $m=2$ in equation (1), yielding the special equation

$$
y^{\prime \prime}+y \exp \left(y^{2}-x\right)=0, \quad x>0 .
$$

We note that (19) is a special case of equation (6) which satisfies assumption (a) of Theorem 1 but fails to satisfy assumption (b). Again we define the Pohozaev's energy function in this case by

$$
H(y(x))=x y^{\prime 2}+x \exp \left(y^{2}-x\right)-y y^{\prime}
$$

which is a special case of (9). Similarly, $E(y(x))=y^{\prime 2}+\exp \left(y^{2}-x\right)$ satisfies $E^{\prime}(y(x))=$ $-\exp \left(y^{2}-x\right) \leq 0$. Hence $E(y(x)) \leq E\left(y\left(x_{0}\right)\right)$ and

$$
\int^{\infty} \exp \left(y^{2}-x\right) d x<\infty
$$

Differentiating $H(y(x))$, we find

$$
\frac{d}{d x} H(y(x))=\left(y^{2}-x+1\right) \exp \left(y^{2}-x\right),
$$

and writing $H(x)$ instead of $H(y(x))$ for short, we have

$$
H(x)-H\left(x_{0}\right)=\int_{x_{0}}^{x}\left(y^{2}-s+1\right) \exp \left(y^{2}-s\right) d s,
$$

which either converges or diverges to $-\infty$. We now rewrite $H(x)$ as

$$
H(x)=x\left(y^{\prime}-\frac{y}{2 \sqrt{x}}\right)^{2}+x \exp \left(y^{2}-x\right)-\frac{y^{2}}{4 x} \geq-\frac{y^{2}}{4 x} .
$$

Note that $y^{2}-x \leq M$ implies $y^{2} \leq k x$ for some $k>0$, so (23) implies $H(x) \geq-k$, and the integral in (22) cannot diverge to $-\infty$, so it must converge to a finite limit, as does $H(y(x))$. Suppose that $y(x)$ is nonoscillatory; then repeating the same argument in the proofs of Lemmas 1, 3 and Theorem 1, we obtain from (16) that

$$
\lim _{x \rightarrow \infty} H(y(x))=0 .
$$

To establish the existence of an oscillatory solution of (19), we therefore need to show the existence of a solution of (19) such that its associated Pohozaev's function $H(y(x))$ tends to some positive constant. Our main result is

Theorem 2. There exists a solution $y(x)$ of (19) with $y\left(x_{0}\right), y^{\prime}\left(x_{0}\right)$ suitably chosen such that

$$
H\left(y\left(x_{0}\right)\right)>-\int_{x_{0}}^{\infty}\left(y^{2}-s+1\right) \exp \left(y^{2}-s\right) d s,
$$

in which case $y(x)$ is oscillatory. In particular, if $y\left(x_{0}\right)=0,\left|y^{\prime}\left(x_{0}\right)\right|=1$ where $x_{0} \geq 10$, then $y(x)$ is oscillatory. 
Proof. Suppose a solution $y(x)$ satisfies (25). From (25) and the convergence of the integral in (22), it is clear that

$$
\lim _{x \rightarrow \infty} H(y(x))=L
$$

exists as a positive constant. Such a solution therefore must be oscillatory, for otherwise, a nonoscillatory solution $y(x)$ must satisfy (24).

To show that we can suitably choose initial conditions $y\left(x_{0}\right), y^{\prime}\left(x_{0}\right)$ so that $(25)$ holds, we start with any solution $y(x)$ of $(19)$ and proceed to estimate the integral in $(25)$, which we denote by $I\left(x_{0}\right)$, for short. Consider two disjoint subsets of the semi-infinite interval $\left[x_{0}, \infty\right)$ as follows:

$$
\begin{aligned}
& X_{1}=\left\{x: x \geq x_{0}, y^{2}-x+1 \geq-3 \log x\right\}, \\
& X_{2}=\left\{x: x \geq x_{0}, y^{2}-x+1<-3 \log x\right\},
\end{aligned}
$$

so that $I\left(x_{0}\right)=\int_{X_{1}}+\int_{X_{2}}$. Here $\int_{X_{i}}, i=1,2$, denotes the definite integral on $\left[x_{0}, \infty\right)$ where the integrand is $\left(y^{2}-x+1\right) \exp \left(y^{2}-x\right)$ if $x \in X_{i}$, but otherwise it is zero. Observe that the convergence of the integral in (22) implies, by (20), that $x \exp \left(y^{2}-x\right)$ is bounded, which in turn implies $y^{2}-x \rightarrow-\infty$ as $x \rightarrow \infty$, for otherwise $x \exp \left(y^{2}-x\right)$ will become unbounded. In particular, we have $y^{2}-x+1 \leq 0$.

For $x \in X_{1}, y^{2}-x+1 \geq-3 \log x$, so $y^{2} \geq x-1-3 \log x \geq \frac{1}{2} x$ for $x \geq x_{0}$ if $x_{0}>10$. We now proceed to estimate $\int_{X_{1}}$ as follows:

$$
\begin{aligned}
-\int_{X_{1}}\left(y^{2}-x+1\right) \exp \left(y^{2}-x\right) d x & \leq \int_{X_{1}} 3 \log x \exp \left(y^{2}-x\right) d x \\
& \leq \int_{X_{1}}\left(\frac{3 \log x \sqrt{2}}{\sqrt{x}}\right) y \exp \left(y^{2}-x\right) d x \\
& \leq \int_{x_{0}}^{\infty}\left(\frac{3 \log x \sqrt{2}}{\sqrt{x}}\right) y \exp \left(y^{2}-x\right) d x \\
& \leq \frac{3 \sqrt{2} \log x_{0}}{\sqrt{x_{0}}} \int_{x_{0}}^{\infty}-y^{\prime \prime}(x) d x \\
& =y^{\prime}\left(x_{0}\right) \frac{3 \sqrt{2} \log x_{0}}{\sqrt{x_{0}}} .
\end{aligned}
$$

Similarly, we note that for all $x \geq x_{0}, y^{2}-x+1 \geq-x$, so on $X_{2}$ we have

$$
\begin{aligned}
-\int_{X_{2}}\left(y^{2}-x+1\right) \exp \left(y^{2}-x\right) d x & \leq \int_{X_{1}} x \exp (-3 \log x-1) d x \\
& \leq e^{-1} \int_{x_{0}}^{\infty} x \exp (-3 \log x) d x \\
& =\frac{e^{-1}}{x_{0}} .
\end{aligned}
$$

Combining (26) and (27), we find

$$
-I\left(x_{0}\right) \leq y^{\prime}\left(x_{0}\right) \frac{3 \sqrt{2} \log x_{0}}{\sqrt{x_{0}}}+\frac{e^{-1}}{x_{0}} .
$$


Suppose $y\left(x_{0}\right)=0$ and $y^{\prime}\left(x_{0}\right)=\alpha>0$. For (25) to hold, we require from (28)

$$
x_{0} \alpha^{2}>\alpha \frac{3 \sqrt{2} \log x_{0}}{\sqrt{x_{0}}}+\frac{e^{-1}}{x_{0}} \text {. }
$$

Clearly, if $\alpha=1$, then $x_{0}>10$ would satisfy (29). The proof is complete.

We note in passing that the quadratic in $\alpha$, as given by (29), does have a positive root. This means that our argument is unable to conclude that every solution of (19) with a zero is oscillatory, as is the case of (1) with $m>2$. Thus, the question whether every solution of (19) with a zero is oscillatory remains open.

\section{Nonoscillation for $0<m<2$}

In this section, we re-examine the non-oscillation result of Atkinson and Chen [4] for (1) when $0<m<2$. The proof given in [4] involves proving cases $0<m<1, m=1$, and $1<m<2$, separately. In the last case, $1<m<2$, they first proved that the energy function $E(y(x))$ tends to zero as $x \rightarrow \infty$ and then appealed to a result due to Coffman and the author [6] to deduce nonoscillation. Their proof that

$$
\lim _{x \rightarrow \infty} E(y(x))=0
$$

when $y(x)$ is oscillatory depends on showing that the zeros $\left\{s_{n}\right\}$ of $y^{\prime}(x)$, the derivative of an oscillatory solution, grow asymptotically like $n^{\mu}$ where $\mu=m /(m-1)$. This part of their proof involves intricate estimates on zeros of certain complicated quadratic functions. Fortunately, an appropriate choice of a different test function in the nonoscillation theorem of [6] proves the assertion directly. The test function used by Atkinson and Chen [4] is $t^{1 / m}$, but in fact, a more appropriate choice should be $(t \log t)^{1 / 2}$. We now shall state and prove a nonoscillation result for (6), somewhat different from the original result in [6], and then show how it can be applied to deduce nonoscillation of (1.1) for $0<m<2$.

Theorem 3. Assume that $x \log x a(x) F(\alpha \sqrt{x} \log x)$ is non-increasing for all large values of $x$ and for all $\alpha>0$, where $F(y)=\int_{0}^{y} f(u) d u$. Then (6) is nonoscillatory.

We first show how Theorem 3 implies nonoscillation of (1) for $0<m<2$. Note that $f(y)=y \exp (|y|)^{m}$ implies, as in the case for $m \geq 2$, that

$$
\begin{aligned}
F(|y|) & =\int_{0}^{|y|} s \exp s^{m} d s \\
& =\frac{1}{m}(|y|)^{2-m} e^{|y|^{m}}\left\{1+O\left(|y|^{-m}\right)\right\}
\end{aligned}
$$

for $|y|$ large. Replacing $|y|$ by $\alpha \sqrt{x} \log x$ in (31) and differentiating $x \log x e^{-x}$ $F(\alpha \sqrt{x \log x})$, we find

$$
\begin{aligned}
& \frac{d}{d x}\left\{\sqrt{x} \log x e^{-x} F(\alpha \sqrt{x \log x})\right\}=R(x) \\
& =(-x \log x+\log x+1) e^{-x} F(\alpha \sqrt{x \log x}) \\
& +x \log x e^{-x} \alpha^{2} \sqrt{x \log x} \exp \left(\alpha^{m} x^{m / 2}(\log x)^{m / 2}\right) \\
& \quad \times \frac{1}{2 \sqrt{x}}\left\{\sqrt{\log x}+\frac{1}{\sqrt{\log x}}\right\} .
\end{aligned}
$$


Using (17) in (32), we obtain

$$
\begin{aligned}
R(x) \sim e^{-x} & \exp \left(\alpha^{m} x^{m / 2}(\log x)^{m / 2}\right) \\
\times & \left\{\frac{1}{m} \alpha^{2-m} x^{1-m / 2}(\log x)^{1-m / 2}(-x \log x+\log x+1)\right. \\
& \left.\times \frac{\alpha^{2} x}{2}\left[(\log x)^{2}+\log x\right]\right\} .
\end{aligned}
$$

Note that in (33), the negative leading term $x^{2-m / 2}(\log x)^{2-m / 2}$ dominates the positive leading term $x(\log x)^{2}$ when $0<m<2$ for $x$ sufficiently large. We conclude that $R(x) \leq 0$ for $x$ large in this case; hence an application of Theorem 3 yields nonoscillation of $(1)$ when $0<m<2$.

We now return to the proof of Theorem 3 and begin by comparing $y(x)$ with $\sqrt{x \log x}$. For a solution $y(x)$ of $(6)$, we define

$$
G(y(x))=x^{2}(\log x)^{2}\left[\frac{d}{d x} \frac{y}{\sqrt{x \log x}}\right]^{2}+\frac{1}{4 x}\left(\log x-\frac{1}{\log x}\right) y^{2}+2 x \log x a(x) F(y)
$$

and use the (6) to compute

where

$$
\frac{d}{d x} G(y(x))=-\frac{1}{2 x^{2}} y^{2}+Q(y, x)
$$

$$
Q(y, x)=\left.2 \frac{\partial}{\partial \sigma}(\sigma \log \sigma a(\sigma) F(\sqrt{\sigma \log \sigma} y(x)))\right|_{\sigma=x} .
$$

The assumption of Theorem 3 implies $Q(y, x) \leq 0$, so for every solution $y(x)$ of (6), we have $\frac{d}{d x} G(y(x)) \leq 0$. In particular, if $y\left(x_{n}\right)=0$, then $G\left(y\left(x_{n}\right)\right)=x_{n} \log x_{n}{y^{\prime}}^{2}\left(x_{n}\right)$ is non-increasing in $n$. Next, if $y(x)$ is an oscillatory solution of (6), then we can determine a uniform bound over the integral $\int_{x_{n}}^{s_{n}} y^{\prime 2}(x) d x$ where $s_{n}$ is a zero of $y^{\prime}(x)$ satisfying $x_{n}<s_{n}<x_{n+1}$. We need the following lemma.

Lemma. Let $y(x)$ be an oscillatory solution of (6) and $y\left(x_{n}\right)=y^{\prime}\left(s_{n}\right)=0, y(x)>0$ for $x_{n}<x<s_{n}$. Then there exists a constant $B_{0}$ such that

$$
I_{n}=\int_{x_{n}}^{s_{n}} y^{\prime 2} d x \leq B_{0}
$$

where $B_{0}$ is independent of $n$.

Proof. Since $Q(y, x) \leq 0$, we can integrate (35) from $x_{n}$ to $s_{n}$ and obtain

$$
\frac{1}{2} s_{n}^{-1} y^{2}\left(s_{n}\right)+2 s_{n} \log s_{n} e^{-s_{n}} F\left(y\left(s_{n}\right)\right) \leq x_{n} \log x_{n}{y^{\prime}}^{2}\left(x_{n}\right)-\frac{1}{2} \int_{x_{n}}^{s_{n}} \frac{y^{2}}{x^{2}} d x
$$

Rewrite the last integral in (37) as

$$
-\frac{1}{2} \int_{x_{n}}^{s_{n}} \frac{y^{2}}{x^{2}} d x=\frac{y^{2}\left(s_{n}\right)}{2 s_{n}}-\int_{x_{n}}^{s_{n}} \frac{y y^{\prime}}{x} d x
$$

Combining the above with (37), we find

$$
\int_{x_{n}}^{s_{n}} \frac{y y^{\prime}}{x} d x \leq x_{n} \log x_{n}{y^{\prime 2}}^{2}\left(x_{n}\right) \leq x_{0} \log x_{0}{y^{\prime}}^{2}\left(x_{0}\right)=B_{1} .
$$


Note that $\left(x y^{\prime}-y\right)^{\prime} \leq 0$ implies $x y^{\prime}-y \leq x_{n} y^{\prime}\left(x_{n}\right)$ for $x \geq x_{n}$, so

$$
\begin{aligned}
I_{n}=\int_{x_{n}}^{s_{n}} y^{\prime 2} d x & \leq \int_{x_{n}}^{s_{n}} y^{\prime}\left(\frac{y+x_{n} y^{\prime}\left(x_{n}\right)}{x}\right) d x \\
& \leq B_{1}+x_{n} y^{\prime}\left(x_{n}\right) \int_{x_{n}}^{s_{n}} \frac{y^{\prime}}{x} d x .
\end{aligned}
$$

Now we have by the Schwarz inequality

$$
\left(\int_{x_{n}}^{s_{n}} \frac{y^{\prime}}{x} d x\right)^{2} \leq \int_{x_{n}}^{s_{n}} y^{\prime 2} d x \int_{x_{n}}^{s_{n}} \frac{1}{x^{2}} d x \leq I_{n} \frac{1}{x_{n}}
$$

hence (39) becomes

$$
\begin{aligned}
I_{n} & \leq B_{1}+x_{n} y^{\prime}\left(x_{n}\right) \frac{I_{n}^{1 / 2}}{\sqrt{x_{n}}} \\
& \leq B_{1}+\frac{\sqrt{B_{1}}}{\sqrt{\log x_{0}}} I_{n}^{1 / 2},
\end{aligned}
$$

from which it follows that $I_{n} \leq B_{0}$ where $B_{0}$ is a constant independent of $n$ (clearly dependent on $x_{0}$ ).

For $x \in\left(x_{n}, s_{n}\right)$ on which $y^{\prime}(x)>0$, we have by (36)

$$
|y(x)| \leq \int_{x_{n}}^{x}\left|y^{\prime}(x)\right| d x \leq I_{n}^{1 / 2}\left(x-x_{n}\right)^{1 / 2} \leq B_{0}^{1 / 2} x^{1 / 2} .
$$

From concavity of $y(x)$ between its zeros, and the fact that $f(y)$ is odd in $y$, we conclude from (40) that $|y(x)| \leq B_{0}^{1 / 2} \sqrt{x}$.

Since we assume $u^{-1} f(u)$ is nondecreasing in $u$, then

$$
p(x)=\frac{a(x) f(y)}{y(x)} \leq \frac{a(x) f\left(B_{0}^{1 / 2} \sqrt{x}\right)}{B_{0}^{1 / 2} \sqrt{x}} .
$$

Observe that for each $\alpha>0$ and $\psi>\varphi$, we have by convexity of $F(u)$

$$
F(\alpha \psi)-F(\alpha \varphi) \geq \alpha(\psi-\varphi) f(\alpha \varphi) .
$$

Letting $\varphi=\sqrt{x}$ and $\psi=\sqrt{x \log x}$ in (42), we find

$$
(x \log x)^{-1 / 2} F(\alpha \sqrt{x \log x}) \geq \alpha f(\alpha \sqrt{x}) .
$$

Denote $B_{0}^{1 / 2}=\alpha$ and substitute (43) in (41); then we obtain

$$
\begin{aligned}
p(x) & \leq \frac{a(x) f(\alpha \sqrt{x})}{\alpha \sqrt{x}} \\
& \leq \alpha^{-2} a(x)(x \log x)^{-1 / 2} \frac{F(\alpha \sqrt{x \log x})}{\sqrt{x}} .
\end{aligned}
$$

Since $a(x) x \log x F(\alpha \sqrt{x \log x})$ is nonincreasing, say bounded by $K$, we deduce from (44) that

$$
p(x) \leq \frac{K \alpha^{-2}(x \log x)^{-3 / 2}}{\sqrt{x}}=o\left(\frac{1}{x^{2}}\right) \leq \frac{1}{4 x^{2}}
$$


Now $y(x)$ satisfies the linear equation $z^{\prime \prime}+p(x) z=0$. Using (45) and an application of Sturm's Comparison Theorem, we conclude that $y(x)$ is nonoscillatory. This completes the proof of Theorem 3 .

\section{References}

1. F. V. Atkinson, On second order nonlinear oscillations, Pacific J. Math. 5 (1995), 643-647.

2. F. V. Atkinson and L. A. Peletier, Ground states and Dirichlet problems for $-\Delta u=f(u)$ and the Emden-Fowler equation, Arch. Rational Mech. Anal. 93 (1986), 103-127.

3. _ Ground states and Dirichlet problems for $-\Delta u=f(u)$ in $\mathbf{R}^{2}$, Arch. Rational Mech. Anal. 96 (1986), 147-165.

4. F. V. Atkinson and S. Chen, On the nonoscillation of an Emden-Fowler equation, AMS Conference Proceedings 8 (1987), 43-55.

5. C. V. Coffman and J. S. W. Wong, On a second order nonlinear oscillation problem, Tran. Amer. Math. Soc. 147 (1970), 357-366.

6. - Oscillation and nonoscillation theorems of solutions of generalized Emden-Fowler equations, Tran. Amer. Math. Soc. 167 (1972), 399-434.

7. L. H. Erbe and J. S. Muldowney, On the existence of oscillatory solutions to nonlinear differential equations, Annali di Mat. Pura ed Applicata 59 (1976), 23-37.

8. S. P. Hastings, Boundary value problems in one differential equation with a discontinuity, J. Diff. Eqn. 1 (1965), 346-369.

9. S. P. Hastings and J. B. McLeod, An oscillatory differential equation, Methods and Applications of Analysis 3 (1996), 432-446.

10. J. W. Heidel and D. B. Hinton, The existence of oscillatory solutions for a nonlinear differential equation, SIAM J. Math. Anal. 3 (1972), 344-351.

11. M. Jasny, On the existence of an oscillating solution of the nonlinear differential equation of the second order $y^{\prime \prime}+f(x) y^{2 n-1}=0, f(x)>0$, Casopis Pest. Mat. 85 (1960), 78-83 (Russian).

12. J. Kurzweil, A note on oscillatory solution of equation $y^{\prime \prime}+f(x) y^{2 n-1}=0$, Casopis Pest. Mat. 85 (1960), 357-358 (Russian).

13. Z. Nehari, On a class of nonlinear second-order differential equations, Trans. Amer. Math. Soc. 95 (1960), 101-123.

14. _ A nonlinear oscillation theorem, Duke Math. J. 42 (1975), 183-189.

15. J. S. W. Wong, On the generalized Emden-Fowler equation, SIAM Review 17 (1975), 339-360.

Department of Mathematics, City University of Hong Kong, Tat Chee Avenue, Kowloon TONG, Hong Kong 